\title{
Resonator-Less Optical Memory in Nanoparticles
}

\author{
B. F. Soares, M. V. Bashevoy, K. F. MacDonald, F. Jonsson and N. I. Zheludev \\ EPSRC NanoPhotonics Portfolio Centre, School of Physics and Astronomy, University of Southampton, SO17 1BJ, United Kingdom \\ Tel. +44-(0)23-8059 2699, Fax.+44-(0)23-8059 3910 \\ Email:bfs@soton.ac.uk
}

\begin{abstract}
For the first time it is shown that nanoparticles undergoing a light-induced structural transformation are inheritably bistable, and we have demonstrated the complete cycle of all-optical writing, reading and erasing information in gallium nanoparticles.

(C)2005 Optical Society of America
\end{abstract}

OCIS codes: (210.4680) Optical memories; (190.3970) Microparticle nonlinear optics

In this paper we report on the first demonstration of resonator-less all-optical optical memory functionalities with picojoule-per-nanoparticle energy requirements, which is achieved by employing a nonlinearity underpinned by light-induced structural transformations in the nanoparticles.

As the electronic sub-structure of a solid is excited, the maximum effect that a single act of optical absorption can have on the optical properties of the medium occurs either when it knocks the molecule out of the light-matter interaction process, for example by saturating the absorption, or when it 'switches on' the optical response of the molecule, for instance by forcing it into resonance. However, a massive change in the optical properties of a medium can be achieved at considerably smaller energies by changing the structural phase of the material, as recently demonstrated for single nanoparticles [1]. A structural change often requires only a small fraction of the photon energy per molecule. For example, the energy difference between the amorphous form and the solid $\alpha$-phase of gallium is only $10^{-3} \mathrm{eV}$ per atom, but the optical properties of these phases are vastly different - one is a good freeelectron metal and the other is essentially a semiconductor. As illustrated in Fig.1, the dynamic coexistence of structural phases in a nanoparticle, which can be controlled by the absorption of light energy, provides a mechanism for reversibly changing the particle's optical properties at low excitation levels and for bistable 'memory' switching at higher excitation levels.

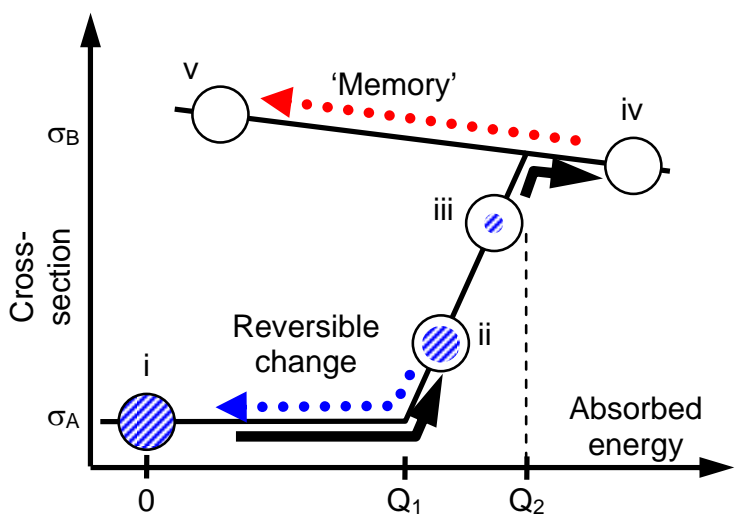

Figure 1. Schematic dependence of optical cross-section on absorbed energy for a nanoparticle undergoing a phase transition (the different phases are represented by the shaded and un-shaded areas). Reversible changes occur in the phase coexistence excitation range between $\mathrm{Q}_{1}$ and $\mathrm{Q}_{2}$; excitation levels above $\mathrm{Q}_{2}$ fully transform the particle to the $\sigma_{\mathrm{B}}$ state, thus providing optical memory functionality.

We demonstrate optical memory functionality using gallium nanoparticles grown by light-assisted self-assembly at the end face of an optical fiber, as illustrated in Fig. 2. A pulsed semiconductor laser operating at a wavelength of $1.5 \mu \mathrm{m}$ was used to generate single control pulses with $1 \mu$ s duration. These pulses switched the gallium nanoparticles from a low reflectivity state ' 0 ' into a high reflectivity state ' 1 '. The state of the bistable nanoparticle film was monitored by a second continuous wave reading laser of low intensity, measuring the reflectivity of the particles. In the absence of the control pulse the reflectivity showed a well-defined hysteresis when cycling the temperature. 


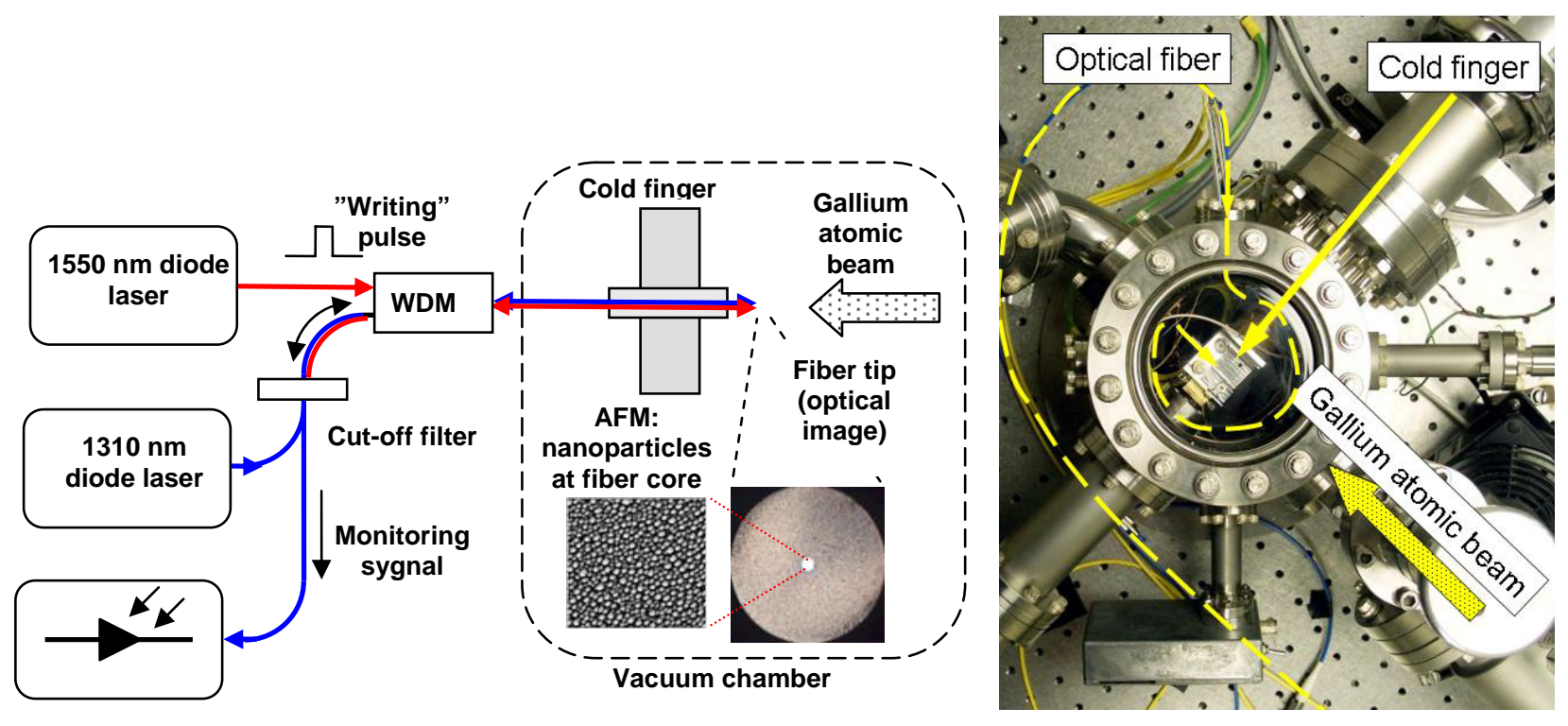

Figure 2. Experimental arrangement for demonstrating optical memory functionality in gallium nanoparticles grown from atomic beam on the tip of optical fibre in vacuum (left) and photograph of the UHV chamber used in the experiment (right). See inset showing AFM image of the nanoparticle film at the fiber core. The optical setup is fully fiberized and bases on low power semiconductor diode lasers.

Holding ‘ 0 ' state

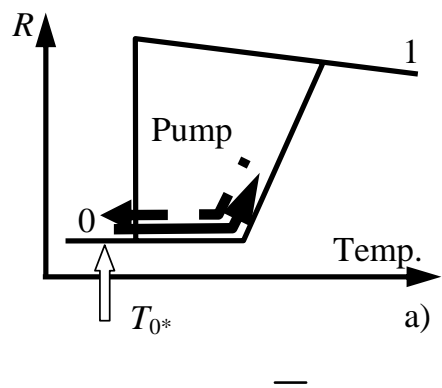

Holding ' 1 ' state

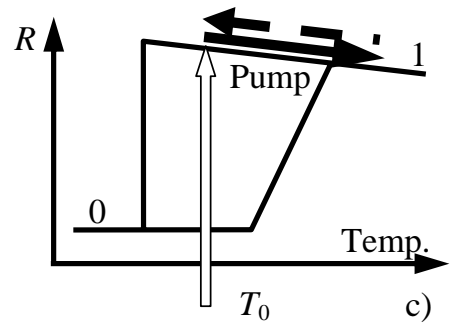

Switch '0' to ' 1 '

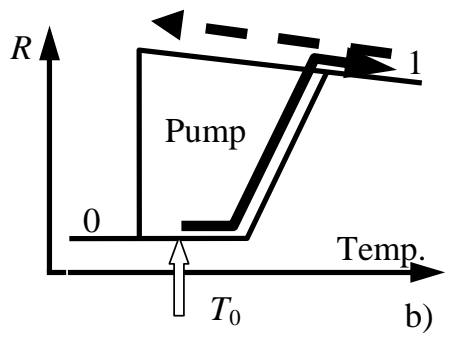

System reset to '0’

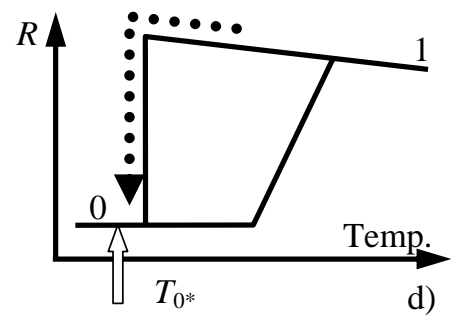

Figure 3. Nanoparticle undergoing light-induced structural transformations as an inherently bistable element. Hollow arrows indicate temperature set points, solid lines show the action of the control optical pulse, and dashed lines show the evolution of the system on termination of the control pulse. The dotted line shows system reset. 
As the temperature of the nanoparticles is set below the lower transition temperature $T_{0^{*}}$ the control pulse causes only a reversible change in the state of the nanoparticles (Fig. 3a). As the temperature $T_{0}$ is increased, the control pulse energy becomes sufficient to fully excite the nanoparticles into a stable high reflectivity phase ' 1 ' (Fig. $3 b$ ), where they remain even after the pulse has passed. The system then stays in state ' 1 ' regardless of the presence of additional control pulses (Fig. 3c). In order to restore state ' 0 ' the temperature of the nanoparticles is decreased below the hysteresis boundary $T_{0^{*}}$ (Fig. 3d), completing the cycle of writing and erasing information to give EPROM functionality. As the temperature set point can be controlled optically by the intensity of the reading beam, in addition to the bias operational temperature, we have also experimentally shown that one can in fact achieve alloptical erase functionality as well, simply by adjusting the reading signal power. Thus, a nanoparticle can constitute an optical memory bit which can be read and erased by a weak probe beam, and written by a single optical control pulse.

[1] B. F. Soares, K. F. MacDonald, V.A. Fedotov, N.I. Zheludev, Nano Letters 5, 10 (2005). 